\title{
Recruitment to a prospective breast conservation trial: Why are so few patients randomised?
}

\author{
W J L Jack, U Chetty, A Rodger
}

\begin{abstract}
Objective-To investigate the rate of recruitment to early breast cancer trials and elucidate the reasons for ineligibility and refusal to participate among patients otherwise suitable for these trials.

Design-Prospective study of one year's cohort of patients referred to a breast unit with special reference to the subgroup suitable for conservation management and to the proportion eligible for and (after informed consent) ultimately randomised within the Scottish early breast cancer trials.

Setting-The breast unit, Longmore Hospital, Edinburgh, during 1988.

Patients-All 3054 patients referred to the breast unit during the year. 324 Found to have invasive breast cancer and 147 initially thought suitable for conservation management.
\end{abstract}

Results - 63 Patients were treated by mastectomy, 19 of whom requested mastectomy rather than conservation management. 84 Patients were excluded from trials, and of the 63 eligible patients, 40 gave informed consent. Most of the 23 patients who refused the trials requested a specific adjuvant treatment after discussion of their management and the trials.

Conclusions-Recruitment to prospective trials in which informed consent is required before randomisation may be slower than predicted because of a high proportion of exclusions and also refusal by patients. Trials may therefore take longer to complete and give distorted results by virtue of the unpredictable nature of the selection of patients.

\section{Introduction}

Much of our knowledge about breast cancer and its management is based on information from clinical trials, but only a very small proportion of all new patients diagnosed each year are treated within trials. Recently it was suggested that in the United States only $2 \%$ of eligible patients with breast cancer are entered into clinical trials.

In Scotland over 2500 new patients with breast cancer are diagnosed each year. Several years ago an assessment based on entry criteria for Medical Research Council Scottish adjuvant trials showed that the rate of entry to the trials was $22 \cdot 2 \%$ of those eligible (H J Stewart, personal communication). The trials therefore took over four times longer to complete than if all eligible patients had been entered.

Recently there has been a more general move towards conservation surgery for early breast cancer. Several prospective trials of different forms of local and systemic management have been reported and others are under way. If these trials have similar problems of low accrual patient selection must be occurring, rendering the results less meaningful when extrapolated to the whole population.
In an attempt to define the proportion of patients suitable for conservation management and to note those entered into the Scottish breast conservation trial a prospective study was set up to analyse one year's cohort of new patients referred to one breast unit. Particular note was made of the reasons why some patients initially deemed suitable for conservation management were finally treated by mastectomy while others were treated by conservation surgery outside the trials - that is, electively.

\section{Method}

In the Lothian region three quarters of patients with breast cancer are referred to the breast unit, which is part of the Edinburgh University department of surgery. Their subsequent treatment is carried out in collaboration with the department of clinical oncology, Western General Hospital, and there is also close liaison with the Scottish Cancer Trials Office (MRC), which administers several breast cancer trials.

All patients are admitted initially to the breast unit at Longmore Hospital, Edinburgh, for staging investigations, including full clinical examination and biochemical and radiological screening. They are seen by the nurse counsellor at the same time and given an explanatory document about clinical trials. After staging is complete treatment options and the appropriate clinical trials are explained to the patient in greater detail. Fully informed consent is obtained from all patients before entry to the prospective randomised trials and patients are given time to discuss all aspects of treatment before decisions are made.

The stage of disease, suitability for trials, and reasons for exclusion are carefully recorded. Patients under 70 who on clinical examination are found to have a single mobile breast cancer not more than $4 \mathrm{~cm}$ maximum diameter are initially considered for conservation treatment. Patients with fixed axillary nodes, supraclavicular nodes, evidence of bilateral disease, or distant metastases are excluded.

The multicentre Scottish breast conservation trial $^{2}$ recruits postmenopausal and node negative premenopausal patients after conservation surgery to the breast and either axillary sampling or clearance, and a second trial (Scottish trial A) running concurrently recruits premenopausal patients with histologically positive nodes who have had either conservation surgery or mastectomy (figure).

The departments of surgery and clinical oncology make every effort to recruit as many patients as possible to these trials after fully informed consent has been obtained.

\section{Results}

During 1988, 3054 patients were referred to the breast unit. Most had benign disease or no abnormality,
Correspondence to: Mr Chetty.

Br Med F 1990;301:83-5 
but 324 patients $(10 \cdot 6 \%)$ were proved to have invasive breast cancer. A subgroup of 147 patients $(45 \cdot 4 \%)$ who were potentially suitable for conservation management were studied further. The figure shows that 84 of these patients ultimately received conservation treatment and 63 mastectomy and that only 40 of the 147 patients $(27 \cdot 2 \%)$ were entered into the conservation trial or trial A.

\section{MASTECTOMY GROUP}

Of the original 147 patients initially deemed suitable for conservation management, 63 were finally treated by mastectomy and therefore were no longer eligible for the conservation trial. Premenopausal patients with positive nodes, however, were still eligible for trial A. Table I gives the reasons for elective mastectomy, which fell into three main groups: patients thought to have more extensive disease after mammography or local excision than apparent clinically (26 cases), patients in whom the cosmetic result of conservation treatment was likely to be poor (12), and patients who requested a mastectomy (19). In the first group 21 of the 26 patients were found to have residual invasive cancer, widespread in situ disease, or multifocal disease present in the mastectomy specimen.

CONSERVATION GROUP

Of the 84 patients who were treated by conservation, 23 did not fulfil the entry criteria for the trials and were

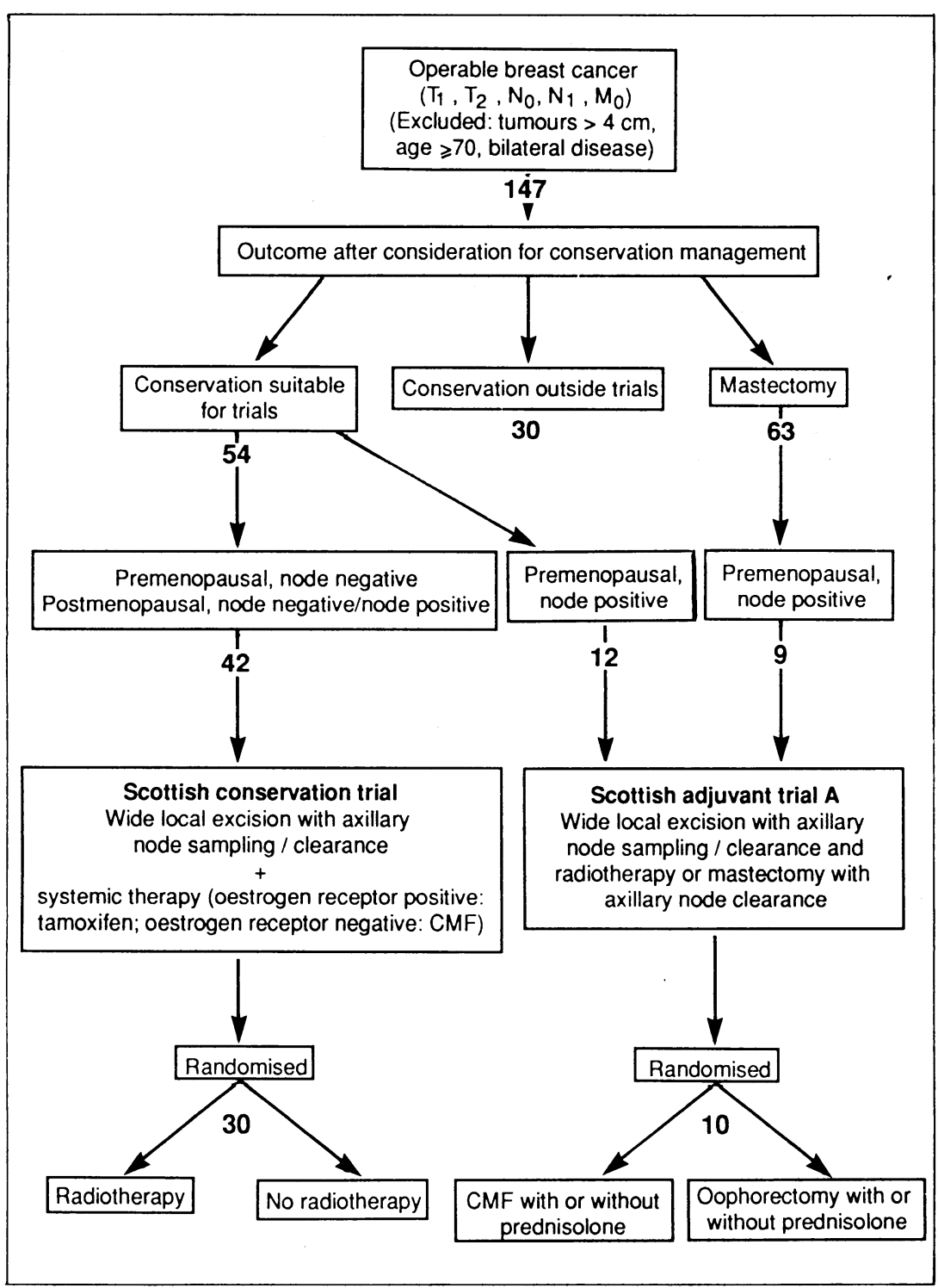

Protocol of randomisation of patients for conservative management or mastectomy. Figures below boxes are numbers of patients. $(C M F=C y c l o p h o s p h a m i d e$, methotrexate, 5-fluorouracil $)$
TABLE I - Reasons for mastectomy

No of patients

\begin{tabular}{lc}
\hline Tumour more extensive than appeared clinically & 26 \\
Incomplete local excision & 15 \\
Widespread in situ disease & 3 \\
Multifocal microcalcification seen on mammography & 3 \\
More than one tumour detected on mammography & 5 \\
Cosmetic result likely to be poor & 12 \\
Patient's request & 19 \\
Other & 6 \\
Suspicion of deep tethering & 1 \\
Poor general health & 3 \\
Previous carcinoma & 2 \\
\hline Total & \multicolumn{2}{|c}{63} \\
\hline
\end{tabular}

TABLE II - Patients treated by conservation but not entered into trials TABLE Potential trial Conservation trial Trial A

\section{Ineligible}

Suspicion of fascial/muscle tethering Poor general health/psychiatric reasons Previous carcinoma

Not randomised for axillary surgery

Postoperative delay

Excluded

Elective radiotherapy/chemotherapy

Elective radiotherapy/chemotherapy
Early breast cancer - no adjuvant therapy

Early breast cancer - no ad
Eligible but not randomised

Patient refused randomisation
Pation but not randomised

Patient refused randomisation
Patient refused chemotherapy

Patient refused chemotherapy
Patient refused oophorectomy

Patient requested chemotherapy

Patient requested oophorectom

Patient requested radiotherapy

Total

\begin{tabular}{|c|c|}
\hline 22 & \\
\hline 6 & 1 \\
\hline 5 & 0 \\
\hline 6 & 0 \\
\hline 4 & - \\
\hline 1 & 0 \\
\hline $2^{4}$ & \\
\hline 2 & $\begin{array}{l}2 \\
1\end{array}$ \\
\hline 12 & \\
\hline 1 & 5 \\
\hline 8 & 0 \\
\hline 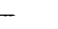 & 1 \\
\hline 0 & 1 \\
\hline 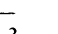 & 3 \\
\hline 3 & - \\
\hline
\end{tabular}

38

therefore ineligible (table II). A further seven patients were excluded electively. Of these, three were thought to have residual disease after wide local excision and were offered postoperative radiotherapy rather than further surgery, one young patient with positive nodes who had had a recent hysterectomy was given elective combination chemotherapy (cyclophosphamide, methotrexate, 5-fluorouracil; CMF) rather than be randomised to possible oophorectomy in trial $\mathrm{A}$, and in three patients further adjuvant therapy after complete excision of a small tumour detected by screening was not deemed justified. Of the remaining patients, 42 were eligible for the conservation trial and 12 for trial A.

\section{TRIALS}

The conservation trial was discussed with the 42 patients who were eligible and informed consent obtained in 30 . Table II lists the reasons for refusal. Twenty one patients were eligible for trial A, and after discussion 10 agreed to participate. Table II gives the reasons for refusal of those managed by conservation (10 cases); one patient who had had a mastectomy refused adjuvant therapy.

Overall, 63 patients were eligible for the two trials and 40 were randomised, and 84 patients $(57 \cdot 1 \%)$ out of the whole group of 147 were ultimately excluded for the reasons detailed above.

\section{Discussion}

A recent paper from Newcastle reported the outcome in a group of patients suitable for conservation treatment who was offered a choice of mastectomy or conservation. Ninety nine $(65 \%)$ out of 153 chose mastectomy. ${ }^{3}$ In our series only 19 of the 103 patients who were offered conservation requested mastectomy. The study from Newcastle covered the period 1979 to 1987 whereas all our patients presented in 1988. The difference may be a reflection of the publicity in the lay press about breast cancer and its management and 
indicate that attitudes among the medical profession and patients are changing.

There has been much discussion about clinical trials and informed consent, ${ }^{4-6}$ and, though we believe that informed consent is mandatory, we accept that a proportion of patients do not enter trials because they may dislike the idea of a random decision being made about treatment or because they refuse or request one part of the random option offered. A further group of patients are excluded because they do not fulfil the entry criteria for the trial, and this group may be larger than forecast at the time the trial was planned. These factors may be the reason why accrual to the Scottish breast conservation trial has been slower than anticipated. The planned total intake was 900 patients, and after four years 420 patients had been entered.

In conclusion more than half of the patients thought initially to be suitable for conservation were excluded from our trials and one third of the remainder refused to take part. Those planning prospective clinical trials should therefore take into account the loss of patients through ineligibility and refusal when predicting the accrual rate and overall duration of any proposed trial and the possible effects of this selection on conclusions drawn from the results.

1 De Vita VT. Breast cancer therapy: exercising all our options. $N$ Engl $\mathrm{f} \mathrm{Med}$ 1989;320:527-9.

Stewart HJ, Prescott RJ, Forrest APM. Conservation therapy of breast cancer. Lancet 1989;ii:168-9.

3 Wilson RG, Hart A, Dawes PJDK. Mastectomy or conservation: the patient's choice. BrMed F 1988;297:1167-9.

4 Brahams D. Medicine and the law. Randomised trials and informed consent. Lancet 1988;ii:1033-4.

Baum M, Zilkha K, Houghton J. Ethics of clinical research: lessons for the future. Br Med F 1989;299:251-3.

6 Brewin $\mathrm{CR}$, Bradley $\mathrm{C}$. Patient preferences and randomised clinical trials. BrMed f 1989:299:313-5.

(Accepted 25 April 1990)

\title{
Interaction between bedding and sleeping position in the sudden infant death syndrome: a population based case-control study
}

\author{
Peter J Fleming, Ruth Gilbert, Yehu Azaz, P Jeremy Berry, Peter T Rudd, Alison Stewart, \\ Elizabeth Hall
}

Abstract

Objective-To determine the relation between sleeping position and quantity of bedding and the risk of sudden unexpected infant death.

Design-A study of all infants dying suddenly and unexpectedly and of two controls matched for age and date with each index case. The parents of control infants were interviewed within 72 hours of the index infant's death. Information was collected on bedding, sleeping position, heating, and recent signs of illness for index and control infants.

Setting-A defined geographical area comprising most of the county of Avon and part of Somerset.

Subjects -72 Infants who had died suddenly and unexpectedly (of whom 67 had died from the sudden infant death syndrome) and 144 control infants.

Results-Compared with the control infants the infants who had died from the sudden infant death syndrome were more likely to have been sleeping prone (relative risk $8.8 ; 95 \%$ confidence interval $7 \cdot 0$ to $11.0 ; \mathrm{p}<0.001$ ), to have been more heavily wrapped (relative risk 1.14 per tog above 8 tog; 1.03 to 1.28 ; $\mathbf{p}<0.05$ ), and to have had the heating on all night (relative risk $2.7 ; 1.4$ to $5.2 ; p<0.01$ ). These differences were less pronounced in the younger infants (less than 70 days) than the older ones. The risk of sudden unexpected death among infants older than 70 days, nursed prone, and with clothing and bedding of total thermal resistance greater than 10 tog was increased by factors of $15 \cdot 1(2.6$ to 89.6$)$ and 25.2 $(3.7$ to 169.0$)$ respectively compared with the risk in infants of the same age nursed supine or on their side and under less than 6 tog of bedding.

Conclusions-Overheating and the prone position are independently associated with an increased risk of sudden unexpected infant death, particularly in infants aged more than $\mathbf{7 0}$ days. Educating parents about appropriate thermal care and sleeping position of infants may help to reduce the incidence of the sudden infant death syndrome.

Correspondence to: Dr Peter Fleming, Department of Child Health, Bristol Maternity Hospital, Bristol BS2 8EG.

Br.Med f 1990;301:85-9 Introduction

The possible role of thermal stress in the aetiology of the sudden infant death syndrome has been suggested by many authors,,$^{1 \cdot 3}$ and Wailoo et al recently showed that many babies are put to bed under excessive amounts of bedding. ${ }^{4}$ The low incidence of the sudden infant death syndrome in Hong Kong, which has a hot, humid climate but where most infants sleep supine, has led to the suggestion that the prone position may be an important risk factor. ${ }^{5}$ Recently, Nelson et al suggested on the basis of a simple model of infant heat balance that infants sleeping in the prone position with an excess of bedding would be more likely to become hyperthermic than infants in the supine position with equal bedding. ${ }^{6}$ We have shown an appreciable rise in oxygen consumption and carbon dioxide production (and hence heat production) between birth and 1 month of age in healthy infants. ${ }^{7}$ This higher metabolic rate is maintained until at least 3 months. We have also shown that raising the environmental temperature around healthy infants aged 3 months or less increases respiratory oscillations, suggesting an effect on the respiratory control system. ${ }^{8}$ Thus infants in this age range, which is the age of peak incidence of the sudden infant death syndrome, would be at increased risk of the consequences of overwrapping. The effects of overwrapping would be likely to be greater at the time of acute viral infection, when the metabolic rate rises, and two studies have shown that many parents in the United Kingdom and in New Zealand respond to infections in their babies by increasing the amount of clothing and bedding..$^{10}$

To investigate the possible interactions between quantity of bedding and sleeping position in normal infants and in infants dying suddenly and unexpectedly we conducted a case-control study of all such infant deaths in a defined part of the counties of Avon and Somerset over 18 months. This study formed part of a prospective investigation of all infant deaths in the county of Avon, which will be reported in full elsewhere.

\section{Methods}

We were notified of all sudden and unexpected deaths of infants (from birth to 1 year) in a defined area comprising most of the county of Avon and part of Somerset. On the day of an infant's death his or her 